\title{
Therapeutic use of hormones on tendinopathies: a narrative review
}

\author{
Michele Abate \\ Matteo Guelfi \\ Andrea Pantalone \\ Daniele Vanni \\ Cosima Schiavone \\ Isabel Andia \\ Vincenzo Salini
}

Department of Medicine and Science of Aging, University "G. d'Annunzio" Chieti - Pescara, Chieti, Italy

\section{Corresponding author:}

Michele Abate

Department of Medicine and Science of Aging, University "G. d'Annunzio" Chieti - Pescara

Via dei Vestini 31

66013 Chieti, Italy

E-mail: m.abate@unich.it

\section{Summary}

Background: Hormones can modify tendon homeostasis, some of them leading to tendon damage, while others are essentials for healing. This narrative review summarizes the current knowledge on the topic, focusing on the hormones normally secreted by endocrine glands.

Methods: A search in PubMed, Web of Knowledge and EMBASE, using the terms tendinopathy or tendon, combined with estrogens, testosterone, thyroid and parathyroid hormones, glucocorticoids and growth hormone, independently, was performed. Relevant articles focusing on the correlation between hormones and tendons, and their therapeutic use in tendinopathies, were selected.

Results: Tendon abnormalities observed in subjects with hyperparathyroidism, hypercortisolism and acromegaly are described. At present, experimental studies and preliminary observations in humans suggest that parathyroid and growth hormones, locally administered, are promising therapeutic tools in specific tendon disorders. Local injections of glucocorticoids are useful in several tendinopathies, exploiting their anti-inflammatory and anti-proliferative properties, but carry the risk of further tendon degeneration and ruptures, due to the detrimental direct effect of glucocorticoids on the tendon structure.

Conclusion: Because tendons injuries are frequent, often with long lasting sequels, it is important to improve our understanding concerning the therapeutic potential of hormones on healing. Level of evidence: IV.

KEY WORDS: estrogens, growth hormone, parathormon, steroids, thyroid, tendinopathy.

\section{Introduction}

Tendons are dynamic structures, characterized by complex metabolic activities. Mechanical loading during exercise is essential to maintain tendon homeostasis. However, when the individual physiologic threshold of loading incidence and magnitude is exceeded, the tendon reaction reverses from favorable towards degenerative. Inflammatory molecules are released and fuel the disease progression. After the failure of the healing process, a smoldering fibrogenesis occurs with matrix turnover and cell activation without normal maturation.

The biological milieu surrounding the tendon components strongly influences the reaction to loading. It has been shown that adverse metabolic situations (diabetes ${ }^{1,2}$, obesity ${ }^{3}$ and hypercholesterolemia ${ }^{4}$ ) may alter the normal tendon response, and favor early degeneration ${ }^{5}$. The frequent association between human tendinopathies and endocrine disorders, as well as experimental data, suggest that also hormones are involved in modifying tendon homeostasis ${ }^{6,7}$. The interest in understanding their mechanism of action strives in developing therapeutic strategies for pathologic tendon conditions.

A full comprehensive review on this topic is not conceivable, thus in this paper we aim to summarize the current knowledge, focusing on the hormones normally secreted by endocrine glands. We performed a search in PubMed, Web of Knowledge and EMBASE using the terms tendinopathy or tendon, combined with estrogens, testosterone, thyroid, parathyroid, glucocorticoids and growth hormone, independently. Studies were deemed relevant if they were published in English and contained original research providing relevant knowledge related to the correlation between hormones and tendons, were selected ${ }^{8}$. Therefore we gathered in vivo and in vitro informations that may provide clues on how to use hormones for tissue healing purposes. 


\section{Clinical evidence}

Several clinical observations suggest that hormonal disorders can be associated with tendinopathies (Tab. I). In some cases the evidence is strong, in others it is less consistent ${ }^{9}$.

There is no evidence that high or low levels of sexual hormones (estrogens, progesterone, testosterone) can be direct cause of tendinopathies. However, an increased incidence of anterior cruciate ligaments $(A C L)$ ruptures has been reported in women involved in sport activities compared to males ${ }^{10}$. Indeed, the sex-linked biomechanical and neuromuscular differences, which contribute to explain this higher incidence $^{5}$, are negligible before puberty, but become evident in the post-pubertal period ${ }^{11}$. However, studies on the adaptations of ligaments and tendons across the menstrual cycle have provided inconclusive data ${ }^{5}$, and recent research suggests that the higher incidence of ACL injuries in females should be attributed to relaxin, which works as a collagenolitic agent. Females, but not males, show specific receptors for this substance, which has been found increased in athletes who underwent tendon ruptures ${ }^{12}$.

The clinical evidence of a relationship between thyroid disorders and tendinopathies is also limited. Stiffness of the hands and knees is frequently observed in patients suffering from hypothyroidism ${ }^{13}$ and an association with shoulder pain has been hypothesized on the basis of epidemiological data ${ }^{9}$. Recently, a case of spontaneous rupture of the long head of the biceps tendon in a woman with hypothyroidism has been reported ${ }^{14}$.

Bilateral tendon ruptures (patellar and Achilles) may occur in patients with secondary or tertiary hyperparathyroidism, the latter condition being characterized by an autonomous parathyroid function, independent of calcium serum concentration. In this case the parathormon $(\mathrm{PTH})$ levels are very high and the clinical pattern is characterized by general osteoporosis, with sub-periosteal bone resorption and resultant weakness of bone-tendon junction ${ }^{15}$. Ultrasound examination in patients submitted to regular hemodialysis allows to identify precociously those at risk, show- ing tenderness during probing, calcific depositions, and increased thickness of Achilles tendon. Tendon ruptures have been reported in subjects with Cushing syndrome or in patients treated chronically with GCs, mainly when associated to fluoroquinolone antibiotics $^{16}$. An epidemiologic survey has shown an increased odds ratio around 3.0 for continous oral GCs, which declined shortly after therapy cessation. Similarly a single short-term high-dose GCs treatment course was sufficient transiently to increase the risk of tendon ruptures ${ }^{17}$.

On the other hand, local GCs injections are frequently performed in subjects with tendinopathies, and show beneficial effects in several cases, because their antiinflammatory and anti-proliferative properties, but carry the risk of further tendon deterioration and ruptures after repeated courses of treatment ${ }^{18}$.

Similarly, in acromegaly, due to long-term exposure to elevated levels of growth hormone $(\mathrm{GH})$ and Insulin-like Growth Factor-1 (IGF-1), besides muscoloskeletal abnormalities (coarsened facial features, growth of hands and feet and soft tissue hypertrophy, articular involvement of the axial and peripheral joints), tendon disorders have been put in evidence ${ }^{19}$. Symptomatic flexor tenosynovitis (trigger finger) is an early manifestation found in $25 \%$ of patients. This condition, which affects the flexor pollicis longus or flexor digitorum tendons, is caused by the entrapment of the affected tendon at first annular pulley, due to proliferation and fibrosis of the sheath and/or to some localized tendon thickening 20 . Also in asymptomatic subjects the thickness of the A1 pulley is higher than in the normal population, as well as the thickness of the heel tendons ${ }^{20}$. The increased tendon thickness is reversible by normalizing GH/IGF-1 excess 20 .

\section{Experimental evidence}

\section{Estrogens}

The clinical observations previously reported have prompted a huge amount of experimental research aimed to explore the intimate mechanisms of action of hormones on tendons (Tab. II).

Table I. Clinical effects of hormonal disorders on tendons.

Estrogens Increased incidence of ACL ruptures in women involved in sport activities (increased production of the collagenolitic agent relaxin).

Thyroid hormones

HyperParathyroidism

Hypercortisolism

Local glucocorticoids injections Beneficial effects in tendinopathies (anti-inflammatory and anti-proliferative properties); Risk of further tendons degeneration and ruptures for repeated injections.

Acromegaly
Stiffness of the hands and knees and shoulder pain in hypothyroidism.

Bilateral tendon ruptures in patients with secondary or tertiary hyperparathyroidism; Calcific depositions and increased thickness of Achilles tendon in patients submitted to hemodialysis.

Tendon ruptures in subjects with Cushing syndrome or treated chronically with GCs.
Trigger finger in $25 \%$ of acromegalic patients (increased thickness of the A1 pulley as well as of the heel tendons in asymptomatic subjects). 
Table II. Effects of hormones on tendons in experimental conditions.

\begin{tabular}{|c|c|}
\hline Estrogens & $\begin{array}{l}\text { Inhibiting effect upon collagen formation (increased expression of MMP-13); } \\
\text { Decreases synthesis of type I collagen, fibronectin and elastin in conditions of estrogen depriva- } \\
\text { tion with a significant lower healing rate in a micro-wound healing model; } \\
\text { Negative influence on the biomechanical tendon properties (both with increased or reduced es- } \\
\text { trogen receptor stimulation). }\end{array}$ \\
\hline Testosterone & Enhanced proliferation of tenocytes. \\
\hline Thyroid hormones & $\begin{array}{l}\text { Enhanced proliferation of tenocytes; } \\
\text { Protective action against apoptosis. }\end{array}$ \\
\hline Parathyroid hormone & $\begin{array}{l}\text { Homeostatic control of fibrochondrocytes at tendon insertion sites; } \\
\text { Production of type I and II (at low mechanical strain) and type X (high mechanical strain) } \\
\text { collagens by fibrochondrocytes. }\end{array}$ \\
\hline Glucocorticoids & $\begin{array}{l}\text { Reduced proliferation and viability of fibroblasts; } \\
\text { Decreased stem cell pool (formation of non-tendinous tissues); } \\
\text { Irreversible senescence in human tenocytes (inhibition of Sirtuin } 1 \text { and activation of the p53/p21 } \\
\text { pathway); } \\
\text { Reduced tendon mechanical properties. }\end{array}$ \\
\hline Growth hormone & $\begin{array}{l}\text { Higher expression for collagen mRNA and collagen fractional synthesis rate in acromegalics } \\
\text { (compared to GH deficient patients); } \\
\text { Higher mRNA expression of collagen types I and III in giant transgenic mice with high circulating } \\
\text { levels of GH and IGF-I (compared to dwarf mice with a disrupted GH receptor gene and to a } \\
\text { wild-type control group); } \\
\text { Increased number of stem cells in vivo and preserved multipotency in vitro; } \\
\text { Inhibition of cell death induced by anoxia. }\end{array}$ \\
\hline
\end{tabular}

The effects of estrogens are not clear. In vitro studies revealed that estradiol has an inhibiting effect upon collagen formation, increasing the expression of matrix metalloproteinase (MMP)-13, which is responsible for tendon collagen degradation ${ }^{10}$. An alternative hypothesis suggests a negative effect of estrogens on the activity of the enzyme lysyl-oxidase, which regulates the addition of lysine and hydroxylysine-based cross-links into collagen fibrils, with ensuing increased ligaments laxity. Further research has shown that estradiol and raloxifene, a specific estrogen receptor modulator, increase the expression of type III collagen and elastin, responsible for tendon elastic properties. These changes can lead to a reduction in tendons' tensile strength because type III collagen fibers are thinner and more flexible than type I fibers $^{21}$.

Paradoxically, similar conclusions have been reached in studies performed in ovariectomized rats, i.e. in conditions of hormonal deprivation ${ }^{22}$. The activity of the extracellular MMP-2 is increased, as well as cell apoptosis both in muscles and tendons ${ }^{23}$. The synthesis of type I collagen, fibronectin and elastin is decreased, and a significantly lower healing rate in a micro-wound healing model has been found ${ }^{22,23}$. In conclusion, both increased or reduced receptor stimulation could influence negatively the biomechanical tendon properties.

\section{Testosterone}

Specific receptors for testosterone have been demonstrated in tendons ${ }^{24}$. In vitro studies have shown that progressive concentrations of dihydrotestosterone enhance the proliferation of tenocytes harvested from healthy supraspinatus tendons ${ }^{25}$. The cultured cells increase in number after 48 and 72 hours after treatment, and acquire a more de-differentiated aspect, becoming more flattened and polygonal with round nuclei, in comparison with control cultures, that show an elongated shape ${ }^{25}$. Therefore, it may be speculated that testosterone regulates lineage determination and differentiation in mesenchymal pluripotent cells resident in tendons ${ }^{25}$.

These results are indirectly supported by the finding that men with low testosterone levels show a significant reduction in both circulating and endothelial progenitor cells, while testosterone replacement therapy induces a significant increase in these progenitors with respect to baseline. This effect can be important during tendon-healing and repair, when active proliferation is required ${ }^{25}$.

\section{Thyroid hormones}

Thyroxine has an important role both in collagen synthesis and matrix metabolism. Hyperthyroidism is accompanied by increased catabolism of both soluble and insoluble collagen, whereas hypothyroidism has opposite effects. Indeed, in subjects with hypothyroidism, glycosaminoglycans deposition in the extracellular matrix explains the pathogenesis of stiff joints and carpal tunnel syndrome and may predispose to tendon calcification ${ }^{13}$.

Thyroid hormone receptors seem to be ubiquitous. Basic observations in vitro have shown that thyroid hormones promote the proliferation, in a dose dependent manner, of tenocytes obtained from tendon biopsies of patients who underwent rotator cuff tears surgery ${ }^{9}$. Moreover, these hormones have a protec- 
tive action against apoptosis induced by serum deprivation, which should be considered an important factor for the failed healing response observed in human tendinopathies ${ }^{9}$. These observations reinforce the idea of a physiological action of thyroid hormones in tendons homeostasis.

\section{Parathormon}

PTH has a relevant role in calcium homeostasis, inducing general osteoporosis, with sub-periosteal bone resorption and resultant weakness of bone-tendon junction ${ }^{15}$.

Parathyroid Hormone-related Protein (PTHrP) belongs to the same PTH gene family, but functions as predominately an autocrine/paracrine regulatory molecule. PTHrP has been identified in the periosteum and tendon insertion sites (entheses), where it controls the biology of fibrochondrocytes ${ }^{26}$, influencing the recruitmnent and/or the activities of underlying bone cell populations ${ }^{27}$. Studies in vitro have shown that cyclic tensile strain increases PTHrP expression. At low mechanical strain, PTHrP induces the production of type I and type II collagens by fibrochondrocytes, while at high mechanical strain it stimulates type $\mathrm{X}$ collagen production, hence tendon mineralization $^{28}$.

\section{Glucocorticoids}

Given the evidence of the healing properties of locally injected GCs in several tendinopathies, however associated to the risk of further tendon deterioration and ruptures in some cases, a plethora of studies has been performed, aiming to elucidate the effects of GCs in isolated tendons, in tendon cell cultures, and in intact animals. Histologically, a loss of collagen organization and an increase of collagen necrosis was observed, followed by a reparative response characterized by an inflammatory cells infiltrate ${ }^{29}$. The proliferation and viability of fibroblasts was also reduced ${ }^{30}$. GCs treatment depletes the stem cell pool and leads to the formation of non-tendinous tissues (e.g. fatty and cartilage-like tissues) ${ }^{31}$. There is in vitro and in vivo evidence that GCs induce irreversible senescence in human tenocytes by inhibition of Sirtuin 1 and activation of the p53/p21 pathway. Tenocytes apoptosis resulted unaffected in some studies, increased in others ${ }^{18}$. Biochemical investigations have shown that collagen synthesis was decreased with an increased ratio of type III to type I collagen ${ }^{32}$. Small numbers of studies demonstrated changes in matrix enzymes (MMPs/TIMPs), proteoglycans, cytokines and other substances including FOX-01 and Sirtuin$1^{32}$. The exposure of human tendon cells to dexamethasone results in a time-dependent reduction of mRNA for Substance $P$, and the induction of Substance $P$ by Interleukin- 1 beta and by cyclic mechanical loading is prevented. This finding could explain why GCs may alleviate pain in tendinopathies. Studies investigating the mechanical properties of tendon after GC injection reported conflicting results. Some Authors observed a decrease in mechanical properties, others an increase, or no significant change ${ }^{33}$.
However, pooling the results, a meta-analysis demonstrated a clear trend towards reduced mechanical properties in tendon after GC injections ${ }^{18}$.

\section{GH/IGF-1 system}

A plethora of experimental data emphasizes the concept that the GH/IGF-1 system is crucial in maintaining muscle-tendon homeostasis. In a study the collagen mRNA expression and collagen protein fractional synthesis rate (FSR) was evaluated locally, in muscles and tendons, by means of microdialysis technique, in acromegalics, relative to $\mathrm{GH}$ deficient patients $^{34}$. A higher expression for collagen and IGF1 mRNA was found in acromegalics. Moreover, there was a tendency towards a higher collagen protein FSR and a smaller collagen fibril diameter in acromegalics in comparison to $\mathrm{GH}$ deficient patients.

The conclusions coming by human studies are supported by animal experiments. Giant transgenic (GT) mice, with high circulating levels of $\mathrm{GH}$ and IGF-I, and dwarf mice with a disrupted $\mathrm{GH}$ receptor gene (GHR-/-), leading to GH resistance and low circulating IGF-I, were compared to a wild-type control group (CTRL). GHR-/- mice had significantly lower collagen fibril volume fraction in Achilles tendon, as well as decreased mRNA expression of IGF-I isoforms and collagen types I and III in muscle compared to CTRL. In contrast, the mRNA expression of IGF-I isoforms and collagens in GT mice was generally high in both tendon and muscle compared to controls. Mean collagen fibril diameter was significantly decreased with both high and low GH/IGF-I signaling, but the GHR-/- mouse tendons were most severely affected with a total loss of the normal bimodal diameter distribution ${ }^{35}$.

$\mathrm{GH}$ plus resistance exercise attenuate structural changes in rat myotendinous junctions resulting from chronic unloading ${ }^{36}$. In addition, GH/IGF-I axis increases the number of stem cells in vivo ${ }^{37}$, and preserves their multipotency in vitro. In addition, the cell death induced by anoxia is inhibited by the addition of IGF-1 through activation of PI3K signaling, suggesting that IGF-1 can be considered as a survival factor $^{38}$. The controversy about whether anabolic tendon adaptations are due to the systemic increase or to local IGF production, or to both, is topic of current research and discussion. Indeed the GH/IGF-1 effects are mediated through endocrine as well as paracrine/ autocrine mechanisms. This system includes the binding proteins, namely $\mathrm{GH}$ binding proteins and IGF-1 binding proteins (IGFBP) ${ }^{39}$. In fact, increased levels of circulating IGF-1 do not always entail biological actions at the local tendon level since only free IGF and binary complexes can leave the blood stream. In fact, most IGF (70-80\%) circulates as ternary complexes composed by IGF-I, IGFBP3 and Acid Labile Subunit. These complexes are too big to leave the blood stream. Only the free form (less than $2 \%$ of circulating IGF-1) or binary compounds (20$25 \%$ of IGF-1 associated to binding proteins, IGFBP1-6) can reach the tendon ${ }^{39}$. Several experiments show that local IGF-1 levels instead of systemic lev- 
els of $\mathrm{GH}$ or IGF-1 are responsible of the stimulation and regulation of collagen synthesis in human tendon tissue. Indeed, studies measuring local IGF-1 response by means of interstitial fluid sampling methodologies have shown that, after exercise, peritendinous values are consistently higher than those of the circulating hormone 40,41 . To add complexity, it is also feasible that the ability for IGF-1 to bind to its receptor in skeletal muscle and tendon is mediated indirectly by the binding proteins. If this is the case, acute exercise can increase the IGF-I receptor binding capacity and affinity to IGF-1, and there may be certain maximal thresholds at which additional circulating IGF-1 is no longer effective.

\section{Therapeutic perspectives}

Because injuries to tendons are frequent and costly, and occur at all ages, mainly in subjects practicing sport activities at professional or amateur level, it is important to improve our understanding concerning the therapeutic potential of hormones in tendon healing. In this section the future perspectives of hormonal therapies are briefly summarized (Tab. III).

The effects of the pill on the incidence of ACL ruptures in females athletes have been evaluated with conflicting results. Indeed, besides studies which report a lower rate of lesions in women who use the pill $^{42}$, in other studies this evidence is lacking ${ }^{43}$. To add complexity, the dosage of estrogens and progesterone vary widely among the pills, and as a consequence the endogenous hormonal levels vary accordingly. Therefore no conclusion can be drawn about the protective effects of contraceptives against $A C L$ injuries.

As far as PTH is concerned, several studies have shown that recombinant PTH (rhPTH) accelerates bone healing and increases chondrocyte recruitment and differentiation ${ }^{44}$. In rats submitted to detachment and immediate repair of the right supraspinatus tendon, using bone tunnel suture fixation, receiving daily subcutaneous injections of $10 \mathrm{mg} / \mathrm{kg}$ of $\mathrm{rhPTH}$, histological analysis revealed, in comparison to controls, at 28 and 56 days, more fibrocartilage, osteoblasts, and blood vessels, and a significantly better collagen fiber orientation 45 . These findings have been confirmed in a rat model of ACL reconstruction with autograph, where intermittently administered PTH has shown to enhance the thickness and micro-architecture of trabecular bone ${ }^{46}$. Unfortunately these promising results did not translate into improved biomechanical properties. More recently, experiments have been performed to evaluate whether rhPTH has healing potential at tendon level. In mice, the deep digital flexor tendon was transected and immediately repaired. PTH (40 mg/kg) was therefore administered, by either subcutaneous or intraperitoneal injections. In comparison to controls, PTH promoted an early formation of reparative tissue associated with an increased expression of extracellular matrix genes, including fibrillar collagens, type I and type III, and fibronectin ${ }^{47}$. Similarly to previous experiments, no significant differences in tensile strength were seen. An incomplete integration/remodeling of the newly formed tissue at the microstructural level, as well the lack of an appropriate mechanical loading and/or the short observation period, are possible reasons that may explain these unsatisfactory results. Therefore, further research is needed to confirm the potential therapeutic effect of PTH in tendon-to-bone or tendon-to-tendon healing.

The use of GCs in the treatment of tendinopathies is an intriguing issue. Intratendinous and peritendinous corticosteroids injections are highly beneficial in trigger finger and De Quervain syndrome, whereas in rotator cuff, patellar and Achilles tendon diseases the results are deceiving and short lasting. Moreover, tendon ruptures have been reported after GC administration. It is evident that the positive effects of GC are probably related to the well-known anti-inflammatory and anti-proliferative properties of these hormones on pathologic tendons, whereas adverse effects must be reported to the detrimental effect of GCs on the tendon structure, when degenerative features are present. Therefore, the boundary between the good and the evil remains uncertain, and caution is required in patients with relapses of chronic overuse tendinopathies. Indeed, in these situations, GC administration, although beneficial in the short term, can worsen tendon degeneration.

Research evaluating the healing properties of $\mathrm{GH} /$

Table III. Therapeutic perspectives.

\begin{tabular}{ll}
\hline $\begin{array}{l}\text { Estrogens+Progesterone } \\
\text { Parathormone }\end{array}$ & $\begin{array}{l}\text { No firm evidence that the pill can reduce the incidence of ACL ruptures in female athletes. } \\
\text { Increased tendon healing in experimental conditions (but not improved biomechanical } \\
\text { properties). }\end{array}$ \\
Glucocorticoids & $\begin{array}{l}\text { Beneficial effects in tendinopathies (but deterioration and possible ruptures after repeated } \\
\text { courses of treatment). }\end{array}$ \\
Increased tenocytes proliferation and collagen production in animals and humans; \\
Increased stem cells number and prevention of their death induced by anoxia; \\
Positive influence on tendon healing in experimental conditions (collagenase-induced tissue \\
atrophy or tendon disruption) and in spontaneous tendinopathies in horses; \\
Increased cross sectional area and biomechanical properties after local injection of \\
recombinant GH in elderly subjects during rehabilitation.
\end{tabular}


IGF-1 is in a more advanced stage. As shown in the previous paragraph, basic studies both in humans and animals have largely demonstrated the positive effects of GH/IGF-1 on tendons (increased tenocytes proliferation and collagen production, increased stem cells number and prevention of their death induced by anoxia). Moreover, studies have shown that this activity is mainly due to local production of IGF rather than to circulating levels of $\mathrm{GH} / \mathrm{IGF}-1$ complex. This finding is relevant because suggests the possibility of IGF-1 delivering in areas of tendon pathology, avoiding the side effects of a systemic administration.

These studies have prompted specific experiments to ascertain whether GH-IGF1 could be an useful tool for tendon healing. The results in animal models are promising. IGF-1 injection promotes tendon and ligament healing after collagenase-induced tissue atrophy or ligament disruption ${ }^{48}$. These findings are substantiated by a retrospective study performed in 40 cases of horse superficial digital flexor tendonitis. The intra-lesional administration of IGF-1 (25 or $50 \mu \mathrm{g} \mathrm{ev}-$ ery other day for 4 or 5 treatments) enhanced healing, as shown by the decrease of ultrasonographic lesions severity, although not to the point to return the horses to sport activities ${ }^{48}$.

Several human studies confirm animal experiments. Human tendon cells cultured in 3D with IGF-I supplementation showed an increased gene expression for collagen, tenomodulin and scleraxis ${ }^{49}$. Healthy humans, who receive either weeks of $\mathrm{GH}$ administration or acute injection of IGF-1, demonstrate increased expression and synthesis of collagen in muscle and tendon $^{32}$. The local injections of recombinant IGF-1 into the patella tendon increased the collagen FSR and procollagen type I N-terminal propeptide (a marker for type I collagen synthesis) in the peritendinous fluid of healthy individuals ${ }^{50}$. Not only IGF-1 but also $\mathrm{GH}$ provides positive results. In a randomized study, two injections of $\mathrm{GH}$ in the patellar tendon of ageing subjects increased collagen synthesis rate 3-4 hours after the second injection, and showed a tendency to higher collagen FSR. Systemic IGF-1 remained unchanged, but interstitial IGF-I increased in GH treated tendons compared with saline treated tendons. This confirms that GH stimulatory effects on tendinous collagen synthesis involves local IGF-1 production ${ }^{50}$. Besides these observations, patients with Ehlers-Danlos syndrome (an inherited connective tissue disorder), treated with $1 \mathrm{mg}$ of IGF-1 injected in the patellar tendon, showed an increase in the protein synthesis rate in comparison with the controlateral tendon used as control ${ }^{35}$.

On the other hand, exercise leads to activation of the systemic GH/IGF-1 axis ${ }^{39}$. Engineered ligaments treated with serum obtained from young healthy men after exercise resulted in more collagen and improved tensile strength in comparison to those treated with serum from resting men. In synthesis, results obtained in different experimental conditions lead to the concordant conclusion that GH/IGF-1 supplementation has positive effects which can favour tendon healing in humans.
Especially elderly people, characterized by an agedependent decline in the GH/IGF-1 activity, may benefit from local GH-IGF-1 administration. It is well known that elderly individuals show lower content of structural proteins in tendons, reduced magnetic resonance imaging tendon signal intensities, and an increase in tendon cross-links due to advanced glycation end products deposition. The GH/IGF-1 axis seems to be important for the maintenance of the structural proteins within these tissues. To assess whether $\mathrm{GH}$ administration could hasten rehabilitation, aged individuals had one leg immobilized two weeks, followed by 6 weeks of retraining. Therefore, the legs immobilized and retrained were divided in two groups, the first receiving daily injections of recombinant $\mathrm{GH}$, and the second saline solution as placebo. Several properties of the patellar tendon, including the cross sectional area, and biomechanical properties were measured, and the ratios type I/III and IGF-1 levels were evaluated in tendon biopsies. The group receiving $\mathrm{GH}$ injections showed an increase of patellar tendon cross sectional area and stiffness, associated to an increase of IGF-1. From these data it may be inferred that local GH administration may be useful to improve healing in patients during rehabilitation of tendon and ligament injuries and postsurgery ${ }^{51}$.

In conclusion, the relationships between hormones and tendons are complex and not fully understood. However, several experimental data suggest that some hormones (mainly GH/IGF-1) may offer significant advances in addition to present conservative treatments for human tendon diseases. A crucial point is to ascertain whether the local administration can be therapeutical, to avoid systemic interactions and undesirable effects. Because tendons injuries are frequent, often with long lasting sequels, this promising therapeutic approach deserves further clinical research.

\section{Conflict of interest}

All the Authors have no disclosure.

\section{References}

1. Boivin GP, Elenes EY, Schultze AK, Chodavarapu H, Hunter $\mathrm{SA}$, Elased KM. Biomechanical properties and histology of $\mathrm{db} / \mathrm{db}$ diabetic mouse Achilles tendon. Muscles Ligaments Tendons J. 2014;4(3):280-284.

2. Snedeker JG, Gautieri A. The role of collagen crosslinks in ageing and diabetes - the good, the bad, and the ugly. Muscles Ligaments Tendons J. 2014; 4(3):303-308.

3. Abate M. How obesity modifies tendons (implications for athletic activities). Muscles Ligaments Tendons J. 2014;4(3):298302.

4. Hast MW, Abboud JA, Soslowsky LJ. Exploring the role of hypercholesterolemia in tendon health and repair. Muscles Ligaments Tendons J. 2014;4(3):275-279.

5. Abate M, Schiavone C, Salini V, Andia I. Occurrence of tendon pathologies in metabolic disorders. Rheumatology (Oxford). 2013;52:599-608. 
6. Frizziero A, Vittadini F, Gasparre G, Masiero S. Impact of oestrogen deficiency and aging on tendon: concise review. Muscles Ligaments Tendons J. 2014;4(3):324-328.

7. Galdiero M, Auriemma RS, Pivonello R, Colao A. Cushing, acromegaly, GH deficiency and tendons. Muscles Ligaments Tendons J. 2014;4(3):329-332.

8. Padulo J, Oliva F, Frizziero A, Maffulli N. Muscles, Ligaments and Tendons Journal - Basic principles and recommendations in clinical and field Science Research: 2016 Update. MLTJ. 2016;6:(1)1-5.

9. Oliva F, Piccirilli E, Berardi AC, Frizziero A, Tarantino U, Maffulli $\mathrm{N}$. Hormones and tendinopathies: the current evidence. $\mathrm{Br}$ Med Bull. 2016;117(1):39-58.

10. Slauterbeck JR, Fuzie SF, Smith MP, et al. The Menstrual Cycle, Sex Hormones, and Anterior Cruciate Ligament Injury. J Athl Train. 2002;37(3):275-278.

11. Andrish JT. Anterior cruciate ligament injuries in the skeletally immature patient. Am J Orthop (Belle Mead NJ). 2001;30:103110.

12. Dehghan F, Haerian BS, Muniandy S, Yusof A, Dragoo JL, Salleh N. The effect of relaxin on the musculoskeletal system. Scand J Med Sci Sports. 2014;24(4):e220-229.

13. Harvie P, Pollard TCB, Carr AJ. Calcific tendinitis: Natural history and association with endocrine disorders. J Shoulder Elbow Surg. 2007;16:169-173.

14. Pantazis K, Roupas ND, Panagopoulos A, Theodoraki S, Tsintoni A, Kyriazopoulou V. Spontaneous rupture of the long head of the biceps tendon in a woman with hypothyroidism: a case report. J Med Case Rep. 2016;10(1):2.

15. Shiota E, Tsuchiya K, Yamaoka K, Kawano O. Spontaneous major tendon ruptures in patients receiving long-term hemodialysis. Clin Orthop Relat Res. 2002;(394):236-242.

16. Claessen FM, de Vos RJ, Reijman M, Meuffels DE. Predictors of primary Achilles tendon ruptures. Sports Med. 2014;44(9): 1241-1259.

17. Spoendlin J, Meier C, Jick SS, Meier CR. Oral and inhaled glucocorticoid use and risk of Achilles or biceps tendon rupture: a population-based case-control study. Ann Med. 2015;47 (6):492-498.

18. Dean BJ, Lostis E, Oakley T, Rombach I, Morrey ME, Carr AJ The risks and benefits of glucocorticoid treatment for tendinopathy: a systematic review of the effects of local glucocorticoid on tendon. Semin Arthritis Rheum. 2014;43(4):570576.

19. Colao A, Marzullo P, Vallone G, et al. Ultrasonographic evidence of joint thickening reversibility in acromegalic patients treated with lanreotide for 12 months. Clin Endocrinol (Oxf). 1999;51(5):611-618.

20. Tagliafico A, Resmini E, van Holsbeeck MT, et al. Sonographic depiction of trigger fingers in acromegaly. J Ultrasound Med. 2009;28(11):1441-1446.

21. Irie T, Takahata M, Majima T, et al. Effect of selective estrogen receptor modulator/raloxifene analogue on proliferation and collagen metabolism of tendon fibroblast. Connect Tissue Res. 2010;51:179-187.

22. Circi E, Akpinar S, Balcik C, et al. Biomechanical and histological comparison of the influence of oestrogen deficient state on tendon healing potential in rats. Int Orthop. 2009;33(5):14611466.

23. Aydin $\mathrm{A}$, Kenar $\mathrm{H}$, Atmaca $\mathrm{H}$, et al. The short- and long- term effects of estrogen deficiency on apoptosis in musculoskeletal tissues: an experimental animal model study. Arch Iran Med. 2013;16:271-276.

24. Khalkhali-Ellis Z, Handa RJ, Price RH Jr, Adams BD, Callaghan JJ, Hendrix MJ. Androgen receptors in human synoviocytes and androgen regulation of interleukin 1 beta (IL1beta) induced IL-6 production: a link between hypoandrogenicity and rheumatoid arthritis? J Rheumatol. 2002;29
(9):1843-1846.

25. Denaro V, Ruzzini L, Longo UG, et al. Effect of dihydrotestosterone on cultured human tenocytes from intact supraspinatus tendon. Knee Surg Sports Traumatol Arthrosc. 2010;18:971976.

26. Chen X, Macica C, Nasiri A, Judex S, Broadus AE. Mechanical regulation of PTHrP expression in entheses. Bone. 2007;41(5):752-759.

27. Kim YJ, Kim HJ, Im Gl. PTHrP promotes chondrogenesis and suppresses hypertrophy from both bone marrow-derived and adipose tissue-derived MSCs. Biochem Biophys Res Commun. 2008;373:104-108.

28. Han X, Guo L, Wang F, Zhu Q, Yang L. Contribution of PTHrP to mechanical strain-induced fibrochondrogenic differentiation in entheses of Achilles tendon of miniature pigs. J Biomech. 2014;47(10):2406-2414.

29. Akpinar S, Hersekli MA, Demirors H, Tandogan RN, Kayaselcuk $F$. Effects of methylprednisolone and betamethasone injections on the rotator cuff: an experimental study in rats. Adv Ther. 2002;19(4):194-201.

30. Tempfer H, Gehwolf R, Lehner C, et al. Effects of crystalline glucocorticoid triamcinolone acetonide on cultered human supraspinatus tendon cells. Acta Orthop. 2009;80(3):357-362.

31. Zhang J, Keenan C, Wang JH. The effects of dexamethasone on human patellar tendon stem cells: implications for dexamethasone treatment of tendon injury. J Orthop Res. 2013;31 (1):105-110.

32. Muto $\mathrm{T}$, Kokubu $\mathrm{T}$, Mifune $\mathrm{Y}$, et al. Temporary inductions of matrix metalloprotease-3 (MMP-3) expression and cell apoptosis are associated with tendon degeneration or rupture after corticosteroid injection. J Orthop Res. 2014;32(10):12971304.

33. Shapiro PS, Rohde RS, Froimson MI, et al. The effect of local corticosteroid or ketorolac exposure on histologic and biomechanical properties of rabbit tendon and cartilage. Hand (N Y). 2007;2(4):165-172.

34. Doessing S, Holm L, Heinemeier KM, et al. GH and IGF1 levels are positively associated with musculotendinous collagen expression: experiments in acromegalic and GH deficiency patients. Eur J Endocrinol. 2010;163(6):853-862.

35. Nielsen RH, Holm L, Jensen JK, et al. Tendon protein synthesis rate in classic Ehlers-Danlos patients can be stimulated with insulin-like growth factor-I. J Appl Physiol. 2014;117 (7):694-698.

36. Curzi D, Lattanzi D, Ciuffoli S, et al. Growth hormone plus resistance exercise attenuate structural changes in rat myotendinous junctions resulting from chronic unloading. Eur $\mathrm{J}$ Histochem. 2013;57(4):e37.

37. Heinemeier KM, Mackey AL, Doessing S, et al. GH/IGF-I axis and matrix adaptation of the musculotendinous tissue to exercise in humans. Scand J Med Sci Sports. 2012;22(4):e1-7.

38. Scott A, Khan KM, Duronio V. IGF-I activates PKB and prevents anoxic apoptosis in Achilles tendon cells. J Orthop Res. 2005; 23:1219-1225.

39. Gatti R, De Palo EF, Antonelli G, Spinella P. IGF-I/IGFBP system: metabolism outline and physical exercise. J Endocrino Invest. 2012;35(7):699-707.

40. Olesen JL, Heinemeier KM, Gemmer C, et al. Exercise-dependent IGF-I, IGFBPs, and type I collagen changes in human peritendinous connective tissue determined by microdialysis. J Appl Physiol (1985). 2007;102(1):214-220.

41. Nindl BC, Scofield DE, Strohbach CA, et al. IGF-I, IGFBPs, and inflammatory cytokine responses during gender-integrated Israeli Army basic combat training. J Strength Cond Res. 2012;26 Suppl 2:S73-81.

42. Martineau PA, Al-Jassir F, Lenczner E, Burman ML. Effect of the oral contraceptive pill on ligamentous laxity. Clin J Sport Med. 2004;14(5):281-286. 
43. Agel J, Bershadsky B, Arendt EA. Hormonal therapy: ACL and ankle injury. Med Sci Sports Exerc. 2006;38:7-12.

44. Weiss S, Hennig T, Bock R, Steck E, Richter W. Impact of growth factors and PTHrP on early and late chondrogenic differentiation of human mesenchymal stem cells. J Cell Physiol. 2010;223(1):84-93.

45. Hettrich CM, Beamer BS, Bedi A, et al. The effect of rhPTH on the healing of tendon to bone in a rat model. J Orthop Res. 2012;30(5):769-774

46. Bi F, Shi Z, Jiang S, Guo P, Yan S. Intermittently administered parathyroid hormone promotes tendon-bone healing in a rat model. Int J Mol Sci. 2014;15(10):17366-17379.

47. Lee DJ, Southgate RD, Farhat YM, et al. Parathyroid hormone 1-34 enhances extracellular matrix deposition and organization during flexor tendon repair. J Orthop Res. 2015;33(1):1724.
48. Witte TH, Yeager AE, Nixon AJ. Intralesional injection of insulin-like growth factor-I for treatment of superficial digital flexor tendonitis in Thoroughbred racehorses: 40 cases (20002004). J Am Vet Med Assoc. 2011;239:992-997.

49. Herchenhan A, Bayer ML, Eliasson $P$, et al. Insulin-like growth factor I enhances collagen synthesis in engineered human tendon tissue. Growth Horm IGF Res. 2015;25(1):13-19.

50. Vestergaard P, Jørgensen JO, Olesen JL, et al. Local administration of growth hormone stimulates tendon collagen synthesis in elderly men. J Appl Physiol (1985). 2012;113(9):14321438.

51. Boesen AP, Dideriksen K, Couppé C, et al. Effect of growth hormone on aging connective tissue in muscle and tendon: gene expression, morphology, and function following immobilization and rehabilitation. J Appl Physiol (1985). 2014;116 (2):192-203 